Irish Math. Soc. Bulletin

Number 77, Summer 2016, 35-43

ISSN 0791-5578

\title{
A LEISURELY ELEMENTARY TREATMENT OF STIRLING'S FORMULA
}

\author{
FINBARR HOLLAND
}

\begin{abstract}
A self-contained account of Stirling's formula for $n$ ! is presented that is based on definitions of the constants $e$ and $\pi$ that appear in it, and uses only the rudiments of the analysis of the convergence of numerical sequences, infinite series, and infinite products.
\end{abstract}

\section{INTRODUCTION}

Stirling's formula tells us that $n$ ! is asymptotically equal to $n^{n} e^{-n} \sqrt{2 \pi n}$, implying that

$$
\lim _{n \rightarrow \infty} \frac{n !}{n^{n+\frac{1}{2}} e^{-n}}=\sqrt{2 \pi} .
$$

This formula is remarkable because it provides an approximation of $n$ ! that consists of a non-integral expression involving the irrational numbers $e$ and $\pi$. In this respect, to somebody seeing it for the first time, it must be just as amazing as Euler's identity linking the numbers $-1, i, e$ and $\pi$ via the equation $e^{i \pi}=-1$.

Over the years, many proofs of equation 11 have been published, and the literature is replete with ones of many different kinds. A sample of these can be found in [2, 4, 15, 6, 7, 8, 9, 10, 11, 12, 13. With one exception - and the present offering is no different in this regard - these articles contain $a d$-hoc proofs of equation 1. Reference [6] is the exception: it is Walter Hayman's most cited article, and his proof illustrates a powerful method for asymptotically estimating the coefficients of a class of power series of analytic functions, termed admissible by him.

2010 Mathematics Subject Classification. 26A09, 26A90.

Key words and phrases. monotonic sequences; central binomial coefficients; Wallis's formula; Tannery's theorem.

Received on 14-1-2016. 
In this note we outline a derivation of equation 1 that could be taught at the end of a first course of analysis that includes a thorough treatment of the theory of convergence of sequences, series and infinite products, and the basic properties of the exponential and trigonometrical functions. It eschews integration completely.

We begin by establishing that there are positive constants $a, b$ such that

$$
a n^{n+\frac{1}{2}} e^{-n} \leq n ! \leq b n^{n+\frac{1}{2}} e^{-n}, n=1,2, \ldots
$$

This is very often all that is needed for many applications. Next, we show that the positive sequence

$$
S_{n}=\frac{n !}{n^{n+\frac{1}{2}} e^{-n}}, n=1,2, \ldots
$$

is decreasing, and so convergent; thereafter, we concentrate on showing that $\sqrt{2 \pi}$ is its limit. This is the heart of the matter, and, to deal with it, we establish Wallis's product formula for $\pi$ from first principles. Along the way we encounter the central binomial coefficients and derive an asymptotic expression for them; whence, as an application, we evaluate $\int_{0}^{\infty} e^{-s^{2}} d s$.

\section{Proof of statement 2}

A rigorous course on sequences and series would surely include a definition of the number $e$, and proofs that the sequences

$$
u_{n}=(1+1 / n)^{n}, v_{n}=(1-1 / n)^{n}, n=1,2, \ldots
$$

are both strictly increasing, and converge to $e$ and $e^{-1}$, respectively, and hence that

$$
\left(1+\frac{1}{n}\right)^{n}<e<\left(1+\frac{1}{n}\right)^{n+1}, n=1,2, \ldots
$$

By considering the Geometric Means of the two lists of positive numbers $u_{1}, u_{2}, \ldots, u_{n}$, and $v_{2}, v_{3}, \ldots, v_{n}$, and utilising the last pair of displayed inequalities, it can be confirmed that the following crude estimates hold for $n$ !, viz.,

$$
(n+1)^{n} e^{-n}<n !<e n^{n+1} e^{-n}, n=1,2, \ldots,
$$

which go someway towards explaining the presence of $e$ in equation 1. In particular, $\frac{1}{\sqrt{n}}<S_{n}<e \sqrt{n}, n=1,2, \ldots$.

To obtain a sharper lower bound for $S_{n}$, we use the power series expansion of the exponential function. 


\section{Lemma 2.1 .}

$$
S_{n}>e^{-1}, n=1,2, \ldots
$$

Proof. Fix $n \geq 1$. Using the power series expansion for $\exp x$ with $x=n$, we have that, for any natural number $m$,

$$
e^{n}>\sum_{k=n}^{n+m} \frac{n^{k}}{k !}=\frac{n^{n}}{n !}\left(1+\sum_{k=1}^{m} \frac{n^{k}}{\prod_{j=1}^{k}(n+j)}\right) .
$$

Now, if $1 \leq k \leq m$,

$$
\begin{aligned}
\frac{n^{k}}{\prod_{j=1}^{k}(n+j)} & =\frac{1}{\prod_{j=1}^{k}\left(1+\frac{j}{n}\right)} \\
& \geq \prod_{j=1}^{k} \exp \left(-\frac{j}{n}\right) \\
& =\exp \left(-\frac{k(k+1)}{2 n}\right) \\
& \geq \exp \left(-\frac{m(m+1)}{2 n}\right) .
\end{aligned}
$$

Hence, for any integer $m \geq 1$,

$$
\frac{e^{n} n !}{n^{n}}>(m+1) \exp \left(-\frac{m(m+1)}{2 n}\right) .
$$

The stated result now follows from this upon setting $m=\lfloor\sqrt{n}\rfloor$.

Theorem 2.2. The sequence $\left(S_{n}\right)$ is strictly monotonic decreasing and converges to a positive number.

Proof. Evidently, $\left(S_{n}\right)$ is strictly monotonic decreasing if and only if

$$
e<\left(1+\frac{1}{n}\right)^{n+\frac{1}{2}}, n=1,2, \ldots
$$

But, $2^{n-1} \leq n !, n=1,2, \ldots$, and so, if $0 \leq x<1$, then

$$
e^{2 x}=1+2 \sum_{n=1}^{\infty} \frac{2^{n-1}}{n !} x^{n} \leq 1+2 \sum_{n=1}^{\infty} x^{n}=\frac{1+x}{1-x},
$$

with equality if and only if $x=0$. Hence, inequality 3 follows on setting $x=1 /(2 n+1)$. Thus, $\left(S_{n}\right)$ is monotonic decreasing. Since it is bounded below by $e^{-1}$, it converges to a positive number as claimed. 


\section{Corollary 2.3.}

$$
e^{-1} n^{n+\frac{1}{2}} e^{-n} \leq n ! \leq e n^{n+\frac{1}{2}} e^{-n}, n=1,2, \ldots
$$

Proof. This is an immediate consequence of Lemma 2.1, and the fact that $S_{n} \leq S_{1}=e, n=1,2, \ldots$.

This result implies statement 2, and suffices in many situations where one is interested in crude but tight estimates for sequences involving the factorial function. For instance, one can use it to obtain good bounds for the sequence of central binomial coefficients $\left(\begin{array}{c}2 n \\ n\end{array}\right), n=1,2, \ldots$. Indeed, it easily follows from statement 4 that

$$
e^{-3} \sqrt{2} 4^{n} n^{-\frac{1}{2}} \leq\left(\begin{array}{c}
2 n \\
n
\end{array}\right) \leq e^{3} \sqrt{2} 4^{n} n^{-\frac{1}{2}}, n=1,2, \ldots
$$

In passing, we note that the sequence $\left(C_{n}\right)$ defined by

$$
C_{n}=\frac{\sqrt{n}\left(\begin{array}{c}
2 n \\
n
\end{array}\right)}{4^{n}}, n=1,2, \ldots
$$

is strictly increasing, and so converges to a positive number, which we denote by $C$; we'll determine its value in Section 4 .

\section{The limit of the Sequence $\left(C_{n} S_{n}\right)$}

It follows from Theorem 2.2 that the limit $S \equiv \lim _{n \rightarrow \infty} S_{n}$ exists, and $S \geq e^{-1}$. We proceed to identify the product $C S$.

Theorem 3.1. $C S=\sqrt{2}$.

Proof. To see this, note that

$$
\begin{aligned}
S_{2 n} & =\frac{(2 n) !}{(2 n)^{2 n} e^{-2 n} \sqrt{2 n}} \\
& =\frac{(2 n) ! \sqrt{n}}{(n !)^{2} 2^{2 n+1 / 2}}\left(\frac{n !}{n^{n} e^{-n} \sqrt{n}}\right)^{2} \\
& =\frac{C_{n} S_{n}^{2}}{\sqrt{2}} .
\end{aligned}
$$

Hence, by the product and subsequence rules for limits of sequences, $S=\frac{C S^{2}}{\sqrt{2}}$, and so $C S=\sqrt{2}$, as claimed.

Thus, once we know $C$, we know $S$, and vice versa. 


\section{The value of $C$}

Starting with the definition of $\pi$ as the smallest positive root of the sine function, in this section we determine $C$ by relating it to the value of the Wallis product.

4.1. The Wallis product. Since, for every positive integer $n$,

$$
\begin{aligned}
C_{n}^{2} & =\left(\frac{(2 n) ! \sqrt{n}}{(n !)^{2} 2^{2 n}}\right)^{2} \\
& =\frac{n}{2 n+1} \frac{((2 n) !)^{2}(2 n+1)}{2^{4 n}(n !)^{4}} \\
& =\frac{n}{2 n+1} \prod_{k=1}^{n}\left(1-\frac{1}{4 k^{2}}\right),
\end{aligned}
$$

we have that

$$
2 C^{2}=\prod_{k=1}^{\infty}\left(1-\frac{1}{4 k^{2}}\right) .
$$

We proceed to relate the value of this infinite product 1 to the limit of another sequence, which comes from a factorisation of $\sin (2 n+1) x$. To obtain this, fix the positive integer $n$, and let $m=2 n+1$, for convenience. Let $\omega=e^{2 \pi i / m}$. Then, for any complex number $z$,

$$
\begin{aligned}
z^{2 m}-1 & =\prod_{r=1}^{m}\left(z^{2}-\omega^{r}\right) \\
& =\left(z^{2}-1\right) \prod_{r=1}^{n}\left(z^{2}-\omega^{r}\right) \prod_{r=n+1}^{2 n}\left(z^{2}-\omega^{r}\right) \\
& =\left(z^{2}-1\right) \prod_{r=1}^{n}\left(z^{2}-\omega^{r}\right)\left(z^{2}-\bar{\omega}^{r}\right) \\
& =\left(z^{2}-1\right) \prod_{r=1}^{n}\left(z^{4}-2 z^{2} \Re \omega^{r}+1\right) .
\end{aligned}
$$

\footnotetext{
${ }^{1}$ Of course, if, at this point, we accept as known that this is $2 / \pi$, we can delete the rest of this section. However, rather than do so, we prefer to prove this result $a b$ initio.
} 
Hence, letting $z=e^{i x}$, where $x$ is any real number, it follows that

$$
\begin{aligned}
\sin (m x) & =2^{n} \sin x \prod_{r=1}^{n}(\cos (2 x)-\cos (2 r \pi / m)) \\
& =2^{2 n} \sin x \prod_{r=1}^{n}\left(\sin ^{2}(r \pi / m)-\sin ^{2} x\right) .
\end{aligned}
$$

In particular, we conclude from this that, first of all,

$$
m=2^{2 n} \prod_{r=1}^{n} \sin ^{2}(r \pi / m)
$$

and thence that

$$
\sin (m x)=m \sin x \prod_{r=1}^{n}\left(1-\frac{\sin ^{2} x}{\sin ^{2}(r \pi / m)}\right) .
$$

Choose $x=\pi / 2 m$, and consider the limiting behaviour of

$$
\frac{1}{m \sin (\pi / 2 m)}=\prod_{r=1}^{n}\left(1-\frac{\sin ^{2}(\pi / 2 m)}{\sin ^{2}(r \pi / m)}\right)
$$

as $n \rightarrow \infty$. Clearly, the sequence on the left side converges to $2 / \pi$. To handle the sequence on the right side, we need a variant of Tannery's theorem [1, 3], tailored to the situation in hand. Since this may not be familiar to many people, we include a proof for completeness.

Lemma 4.1. Suppose $a_{m}(n), m, n=1,2, \ldots$, is a double sequence of complex numbers, with the following properties:

(1) there is a sequence of positive numbers $m_{r}, r=1,2, \ldots$, such that $\prod_{r=1}^{\infty}\left(1+m_{r}\right)$ is convergent, and

$$
\sup _{n \geq 1}\left|a_{r}(n)\right| \leq m_{r}, r=1,2, \ldots ;
$$

(2) for each $m$, the sequence $a_{m}(n), n=1,2, \ldots$, converges to $a_{m}$

(3) the sequence $P_{n}$, defined by

$$
P_{n}=\prod_{m=1}^{n}\left(1+a_{m}(n)\right), n=1,2, \ldots,
$$

converges to $P$, say. 
Then

$$
P=\prod_{m=1}^{\infty}\left(1+a_{m}\right)
$$

Proof. Fix $m$ and let $n>m$. Then

$$
\begin{aligned}
\left|P_{n}-\prod_{r=1}^{m}\left(1+a_{r}(n)\right)\right| & =\left|\prod_{r=1}^{m}\left(1+a_{r}(n)\right)\right|\left|\prod_{r=m+1}^{n}\left(1+a_{r}(n)\right)-1\right| \\
& \leq \prod_{r=1}^{m}\left(1+m_{r}\right)\left(\prod_{r=m+1}^{n}\left(1+m_{r}\right)-1\right) \\
& =Q_{n}-Q_{m}
\end{aligned}
$$

where

$$
Q_{n}=\prod_{r=1}^{n}\left(1+m_{r}\right), n=1,2, \ldots
$$

Under our assumptions, the sequence $Q_{n}$ converges to the infinite product

$$
Q=\prod_{m=1}^{\infty}\left(1+m_{r}\right)
$$

while $\lim _{n \rightarrow \infty} a_{m}(n)=a_{m}$, and $\lim _{n \rightarrow \infty} P_{n}=P$. Hence, letting $n \rightarrow \infty$, we deduce from the inequality just established that

$$
\left|P-\prod_{r=1}^{m}\left(1+a_{r}\right)\right| \leq Q-Q_{m}, m=1,2, \ldots,
$$

whence the result follows.

We apply Lemma 4.1 with

$$
a_{r}(n)=-\frac{\sin ^{2}(\pi / 2 m)}{\sin ^{2}(r \pi / m)}, r=1,2, \ldots, n, n=1,2, \ldots
$$

Since $2 x / \pi \leq \sin x \leq x$, if $0 \leq x \leq \pi / 2$, it follows that

$$
\sup \left\{\left|a_{r}(n)\right|: n=1,2, \ldots\right\} \leq \frac{\pi^{2}}{16 r^{2}}, r=1,2, \ldots
$$

Also

Hence

$$
\lim _{n \rightarrow \infty} a_{r}(n)=-\frac{1}{4 r^{2}}
$$

$$
\lim _{n \rightarrow \infty} \prod_{r=1}^{n}\left(1-\frac{\sin ^{2}(\pi / 2 m)}{\sin ^{2}(r \pi / m)}\right)=\prod_{r=1}^{\infty}\left(1-\frac{1}{4 r^{2}}\right)
$$


Combining our results we may conclude that

$$
2 C^{2}=\prod_{r=1}^{\infty}\left(1-\frac{1}{4 r^{2}}\right)=\frac{2}{\pi},
$$

and so $C=1 / \sqrt{\pi}$. It follows that $S=\sqrt{2 \pi}$.

5. The value of the integral $\int_{0}^{\infty} e^{-s^{2}} d s$

As a quick look at the references reveals, many proofs of equation 1 rely on knowing the value of this integral. Here, we reverse matters and essentially derive its value from equation 1, by identifying it directly with $C \pi / 2$. Conversely, of course, making this identification, we can dispense entirely with the previous section and obtain equation 1 very quickly.

Since $\left(\begin{array}{c}2 n \\ n\end{array}\right)$ is the constant term in the trigonometrical polynomial $\left(e^{i x}+e^{-i x}\right)^{2 n}$, it's clear that

$$
\frac{1}{2 \pi} \int_{-\pi}^{\pi} \cos ^{2 n} x d x=2^{-2 n}\left(\begin{array}{c}
2 n \\
n
\end{array}\right), n=0,1, \ldots
$$

Hence, for $n=1,2, \ldots$,

$$
\begin{aligned}
C_{n} & =\frac{\sqrt{n}\left(\begin{array}{c}
2 n \\
n
\end{array}\right)}{2^{2 n}} \\
& =\frac{\sqrt{n}}{2 \pi} \int_{-\pi}^{\pi} \cos ^{2 n} x d x \\
& =\frac{2 \sqrt{n}}{\pi} \int_{0}^{\pi / 2}\left(1-\sin ^{2} x\right)^{n} d x \\
& =\frac{2 \sqrt{n}}{\pi} \int_{0}^{1}\left(1-t^{2}\right)^{n-\frac{1}{2}} d t \\
& =\frac{2}{\pi} \int_{0}^{\sqrt{n}}\left(1-\frac{s^{2}}{n}\right)^{n-\frac{1}{2}} d s .
\end{aligned}
$$

But, if $0 \leq s \leq \sqrt{n}$, then

$$
\left(1-\frac{s^{2}}{n}\right)^{n-\frac{1}{2}} \leq e^{-\left(n-\frac{1}{2}\right) s^{2} / n}=e^{-s^{2}} e^{s^{2} / 2 n} \leq e^{-s^{2}} \sqrt{e} .
$$

Furthermore,

$$
\lim _{n \rightarrow \infty}\left(1-\frac{s^{2}}{n}\right)^{n-\frac{1}{2}}=e^{-s^{2}}
$$


Hence, by the variant of Tannery's theorem for integrals, or by Lebesgue's dominated convergence theorem,

$$
C=\lim _{n \rightarrow \infty} C_{n}=\frac{2}{\pi} \int_{0}^{\infty} e^{-s^{2}} d s .
$$

\section{REFERENCES}

[1] T. J. I'A. Bromwich: An Introduction to the Theory of Infinite Series (2nd edition), Macmillan, London, 1926.

[2] R. B. Burckel: Stirling's formula via Riemann sums, Coll. Math. J. 27 (2006), 300-307.

[3] E. T. Copson: An Introduction to the Theory of Functions of a Complex Variable, Oxford University Press, 1935.

[4] P. Diaconis, D. Freedman: An elementary proof of Stirling's formula, Amer. Math. Monthly, 93 (1986), 123-125.

[5] William Feller: A direct proof of Stirling's formula, Amer. Math. Monthly, 74 (1967), 1223-1225.

[6] W. K. Hayman: A generalisation of Stirling's formula, J. Reine Angew. Math., 196 (1956), 67-95.

[7] George Marsaglia and John C. W. Marsaglia: A new derivation of Stirling's approximation to n!, Amer. Math. Monthly, 97 (1990), 825-829.

[8] Reinhard Michel: On Stirling's formula, Amer. Math. Monthly, 109 (2002), 388-390.

[9] Thorstein Neuschel: A new proof of Stirling's formula, Amer. Math. Monthly, 121 (2014), 350-352.

[10] J. M. Patin: A very short proof of Stirling's formula, Amer. Math. Monthly, 109 (2002), 388-390.

[11] Herbert Robbins: A remark on Stirling's formula, Amer. Math. Monthly, 62 (1955), 26-29.

[12] Dan Romik: Stirling's approximation for n!: The ultimate short proof?, Amer. Math. Monthly, 107 (2000), 556-557.

[13] G. Rzadkowski: Remarks on the formulae of Stirling and Wallis, Math. Gazette, 81 (1997), 427-431.

Finbarr Holland is professor emeritus at his alma mater University College Cork (UCC), where he received his primary degree in 1961. The following year he was awarded a Travelling Studentship in Mathematical Science, and a master's degree by the National University of Ireland, on receipt of which he studied for his $\mathrm{PhD}$ in Harmonic Analysis at University College, Cardiff, under the supervision of Lionel Cooper. He was awarded this degree by the National University of Wales in 1964. Following a year in Caltech as a research fellow, in 1965 he was appointed to a post at UCC where he has taught ever since. He contributes regularly to the 'Problem Section' in several journals.

(Finbarr Holland) School of Mathematical Sciences, University ColLEGE CORK

E-mail address: f.holland@ucc.ie 